\title{
Experimental Study of the Effects of Wire EDM on the Characteristics of Ferritic Steel, At a Micro-Scale on the Contour Cut Surface
}

\author{
N. Naveed ${ }^{1,2}$ \\ ${ }^{1}$ University of Sunderland, The David Goldman, St Peter's Way, SR6 0DD \\ ${ }^{2}$ The Open University, Walton Hall, Milton Keynes, MK7 6AA \\ United Kingdom \\ Nida.Naveed@sunderland.ac.uk
}

\begin{abstract}
This study, on a micro-scale, of the WEDM cut surfaces of specimens to which the Contour Method of residual stress measurement is being applied provides detailed information about the effects of the cutting process on the surface quality. This is defined by a combination of several parameters: variation in surface contour profile, sub-surface damage and surface texture. Measurements were taken at the start, the middle and at the end of the cut. This study shows that during WEDM cutting, a thin layer, extending to a depth of a few micrometres below the surface of the cut, is transformed. This layer is known as the recast layer. Using controlled-depth etching and X-ray diffraction, it is show that this induces an additional tensile residual stress, parallel to the plane of the cut surface. The WEDM cut surface and sub-surface characteristics are also shown to vary along the length of the cut. Moreover, these micro-scale changes were compared with macro scale residual stress results and provides an indication of the point at which the changes occurred by cutting process can be significantly relative to the macro-scale residual stress in a specimen.
\end{abstract}

Keywords: Wire Electrical Discharge Machine (WEDM), Recast layer, Electro-chemical polishing, Surface roughness, Contour method.

\section{Introduction}

The contour method is a destructive technique for measuring residual stress. It was first proposed in 2000 by Mike Prime [1]. The theory of the contour method is mainly based on Bueckner's elastic superposition principle [2]. The first step is to cut the test components in two along the plane of interest. The cut surfaces deform (deviate from perfect flatness) owing to the relaxation of residual stresses. This deformation is measured and input as the boundary conditions in a finite element analysis to back-calculate a 2-dimensional map of the original residual stresses normal to the plane of the cut. Thus, the contour method mainly involves four steps: specimen cutting, surface contour measurement, data reduction and finite element modelling. The cutting step is the fundamental part of the contour method, because all the raw data from which the results are calculated are, directly obtained from the surfaces so created. Therefore, as a destructive procedure that by definition cannot be repeated, cutting must be conducted with special care, as the quality of the cut surface directly affects all the subsequent steps.

In general, destructive techniques - including the contour method - for measuring residual stresses are based on material removal. However, other material removal techniques such as incremental central hole drilling, deep hole drilling [3], [4], slitting (crack compliance) [5], layer removal, ring coring [4] Sachs boring [6] are not as sensitive as the contour method to the cutting step. In most destructive residual stress measurement techniques, some of the stressed material is removed from the specimen and the resulting deformation in the adjacent material is measured, as a strain. This is often done by monitoring strain gauges attached before any material has been removed. Often, the geometry of the surface where the deformations are to be measured is different from that of the removed material. In these cases there is not a direct relationship between the measured relieved strain and inferred residual stress. The contour method is not subject to this limitation.

The purpose of the present work is to investigate the effects of the cutting process on the characteristics of ferritic steel at a micro-scale on the contour cut surface. The surface form, depth of recast layer, roughness and the depth of residual stresses are measured.

\section{The Contour Method Cutting Assumptions}

It is assumed that any deviation of the newly created cut surfaces has occurred solely because of the elastic relaxation of residual stresses acting normal to the plane of interest prior to the cutting. Therefore, for the cutting processes the following criteria must be fulfilled: a) cutting should be flat at the plane of interest and a minimal quantity of material should be removed; b) the width of any material removed by the cutting process (the kerf) should be constant; c) the cutting process must not induce any stress on the cut surface and does not modify the original residual stresses; d) the cutting process should not induce plastic deformation. In summary, the ideal cut would have zero width, induce no stresses and allow no plasticity at the tip of the cut [7].

The cut must be done in a single pass. This is different from conventional cutting, where multiple cuts can be made in order to achieve the best surface finish. Wire Electric Discharge Machining (WEDM) has previously been identified as the best choice for the cutting step in the contour method [8], [9] as it is capable of producing a cut surface with high precision, low roughness, and high wear resistance in a single pass [10]. It can be used for all electrically conductive materials irrespective of their hardness, material strength, shape and toughness. WEDM is a non-contact machining process; there is no direct contact between the electrode and the work piece during cutting. Consequently, it generates no cutting force, in contrast to other conventional cutting methods. Because very little heat is generated either, it does not deform the cut surface, further reducing mechanical stresses and entirely eliminating vibration problems that can occur with other methods (such as fine wire abrasive cutting) [11].

\section{The Wire EDM Process and Its Effects on the Contour Cut Surface}

WEDM cutting is a thermo-electric process and it is performed by generating a series of electrical sparks between the WEDM wire and the component [12]. Because of the high temperature of the spark, the surface and the sub-surface (up to a few microns of the specimen) are heat-affected and there is a potential for consequent changes in texture and other materials characteristics. The extent to which these could affect residual stress measurements have not yet been fully investigated, although some progress has been made in relating WEDM parameters to the effect on the material condition [13].

When residual stress normal to the plane of the cut is relieved, both cut surfaces will deform either towards or away from each other, depending on whether the stress was compressive or tensile. There is a potential for geometric changes to occur on the cut surface contours in the result of any violation of the cutting assumptions. These introduce artefacts and consequently errors and uncertainties in the residual stress measurements [7], [14].

These artefacts can be classed as either symmetric (since ideally the surfaces are mirror images of one another) or anti-symmetric [7]. In general, not all of the distortion of a cut surface, even in the absence of 
cutting artefacts will be symmetric, for example owing to shear stresses. Asymmetrical artefacts of the cutting process can result from asymmetrical constraint of the specimen during cutting, or the use of curved or crooked cuts.

Errors related to anti-symmetric cutting artefacts are of less concern because they can be removed by averaging the data obtained from contours of both cut surfaces [7], [15]. In contrast, symmetric cutting artefacts can cause significant errors because they cannot be removed in this way. Symmetric errors can be divided into two groups according to whether or not they are dependent on the magnitude of the real residual stress. Symmetric errors dependent on the stress magnitude can be further classified as either elastic bulging or plasticity errors or both combined. An ideal cut in a stress-free body would produce a well-defined surface profile; i.e. both parts of the cut would match exactly and represent perfectly flat mating surfaces. Thus any distortion on the cut surfaces of a specimen originally stress-free can be identified as cutting artefacts.

The WEDM cutting process produces surfaces with an underlying level of roughness. This imposes a fundamental limit on the residual stress length scale resolution of the contour method [16]. Surface roughness can be quantified by the vertical deviations of a real surface from its ideal form, and can be represented by various parameters [17]. In addition to this the cutting process can introduce features such as flared edges, steps, waviness and surface bowing [16], [18]. Flared edges are often seen at wire entry and exit locations, and at the start and end of the cut (i.e. at transition locations). By definition, these sites are places where there is a step discontinuity in the cutting conditions. For example the flushing away of debris by the water jets is more chaotic than within the specimen. This is because the specimen boundaries present discontinuities in the medium surrounding the jets. The turbulence this creates can produce wire vibration and high deformations at these locations. Another sharp transition here is in the electric field associated with the discharge. Greater electrical energy in the discharge pulse in these regions of higher field strength [19], [20] results in a higher erosion rate along the edges. This violates the assumption that a constant width of material is removed. For this reason, practitioners of the contour method often do not report stresses in these regions near the outer surfaces of the specimen.

To deal with this issue and produce reliable near surface stress results, sacrificial layers of material similar to that of the specimen can be attached at the top and bottom faces of the specimen along the plane of the cut as well as at the start and at the end of the cut [21]. Recently, some success has been reported in obtaining accurate near-surface stress results using sacrificial layers for a specimen with a complex geometry [22], [23]. Sharp variations in apparent deformation across the width of the component are called a "step". These cutting artefacts are commonly seen on the cut surface where the cut was performed on a work-piece that has non-uniform thickness (resulting in a sharply changing wire contact length). Such sudden changes in the wire contact length can cause instability of the cutting conditions. Carefully shaped or cast in-situ sacrificial layers can be effectively deployed in such cases. Periodic variations of surface displacement measurements, normally along the cut direction, are termed waviness. This can be caused by local cutting irregularities such as wire vibration and wire drift. These artefacts can be avoided by increasing the wire tension. Sometimes the cut surface is curved. This feature can be categorised as either a "convex bowed" or a "concave bowed" form of cut. In case of the convex bowed form, more material is removed close to the top and bottom, or at the start and end of the cut. The concave bowed form occurs when more material is removed at the mid thickness of the cut. These features can occur due to wire vibration and wire bending during cutting. This is a symmetric cutting artefact, and so cannot be cancelled out by averaging data from the surface contours. Wire instability can occur during the cutting process for several reasons. The electrical spark applies several variable forces upon the wire, such as electrostatic and electrodynamic forces [24]. In addition to this, the flushing pressure, and the formation and evacuation process of debris can also cause wire instabilities. These artefacts can be minimised by adopting appropriate settings for the WEDM cutting. The WEDM cutting parameters and the cutting conditions are machine specific, with each machine offering a library of different, established cut settings. These cut settings are highly dependent on the properties of each specimen, such as its geometry and material type. The optimum settings for specific properties of the specimen can be determined by undertaking stress-free trial cuts [15], [21].

Any non-planar shape present on the surface of the cut can then be identified as an artefact of the WEDM cutting process [14]. These errors can be subtracted from the measured surface contour of the actual cut. In order to follow best practice, this test cut should be performed on stress-free portions of the same material of the same thickness, cut off at a small distance from the original cut surface on the actual test specimen [14], [25], [26].

Elastic bulging artefacts are highly dependent of the stress state within the component. Elastic bulging can be produced as the cutting proceeds and material is being relaxed; the material at the cut tip deforms due to stress relief and reduces the original width of the cut. The size of this effect depends on the stress-relieved state at the tip of the cut relative to the original state of stress. This error can be minimised using secure clamping of the test component. It also depends on the direction of cut and the kerf width. It decreases as the kerf width decreases.

Plasticity at the cut tip is also a source of error in contour measurements. Both plasticity error and bulge error are dependent on the stress state at the cut tip. This can be characterised by the stress intensity factor and any reduction in the severity of the stress at the cut tip would result in reducing the plasticity error and bulge error [27] As the cut progresses, relaxation of that part of the stress field that was acting across the plane of the cut, causes a redistribution of the remaining residual stresses. This can result in an increased stress at the advancing tip of the cut [28]. As the cut length increases the stress concentration becomes more intense and may result in local yielding, and this introduces plasticity errors at the tip of the cut.

In addition to these features, others, such as the formation of a recast layer - or white layer - can also affect the surface profile measurements. During WEDM cutting, the spark vaporizes small quantities of material from the surface, and a proportion of this is immediately re-deposited as the recast layer [29]. During cooling and solidification of that layer, the microstructure and material properties can be altered, both within the newly-formed layer and in the heataffected zone of the parent material [30], [31]. The recast layer damages the cut surface, producing defects such as voids and cracks. It also generates small tensile residual stresses at the cut surfaces [32]. The recast layer increases the surface roughness, makes the surface hard and brittle, and decreases the fatigue strength and corrosion resistance of the material [30]. The features of the contour cut surface are highly dependent on the WEDM cutting conditions (such as the WEDM cutting parameters, debris flushing and the nature of the dielectric fluid) and the material properties of the specimen (such as yield strength, thermal expansion coefficient and thermal conductivity). Materials having a large thermal expansion coefficient, and a low thermal conductivity would be expected to suffer more surface damage resulting from the WEDM process [33]. The form of the residual stress profile and the maximum values of tensile stresses are independent of the discharge energy, whereas the depth from the surface at where the peak tensile stress occurs is energy dependent [34].

This complex combination of possible influences on the profile of the cut surface merits an investigation of their relative importance. The micro-scale investigation reported here is part of a larger study that aims to achieve a full understanding of all the processes contributing to uncertainty in residual stress measurements obtained by the contour method. As mentioned above, the WEDM cut surfaces are mostly greatly affected at the wire entry and wire exit, and at the cut start and end positions (i.e. all the way round the perimeter of the new surfaces created by the cut). These regions were therefore the main focus of this investigation. The depth of the recast layer on the WEDM cut surface was measured using SEM analysis of cross-sections through the cut surfaces. The effect of the recast layer on residual stresses was also investigated, by incremental removal using electro-polishing, and $\mathrm{X}$ ray diffraction measurements at each depth. Nano-indentation tests were performed to determine the sub-surface changes in the materials properties. The surface form and roughness were measured using a 3D confocal microscope. Linear elastic finite element analyses were conducted to interpret the contribution of the surface deformation 
features to the apparent stresses on contour cut surface. The whole topography of the contour cut surface was examined and cut surface data were averaged along the length of the cut (the width of the plate$\mathrm{Y}$ axis). Also, the contour cut surfaces were studied particularly at the start, the middle and at the end portions of the cut.

\section{Test Specimens}

\section{A Ferritic Bead on Plate (Bop)}

A single bead on plate weldment was made using the gas tungsten arc welding process. The substrate plate was made from SA508 grade 3 class 1 steel. The plate was machined to the dimensions of $180 \times 120$ x $20 \mathrm{~mm}$, as shown in Figure 1(a). In order to eliminate machining stresses the plate was stress relieved by furnace post weld heat treatment at $600^{\circ} \mathrm{C}$ prior to welding. Then a $100 \mathrm{~mm}$ long single weld bead was deposited in the longitudinal direction at mid-width of the plate with a Oerlikon SD3 1Ni 1/4 Mo TIG wire filler. The SD3 weld metal yield strength was approximately $10 \%$ overmatched compared with the SA508 steel plate. At $20^{\circ} \mathrm{C}$, the $0.2 \%$ proof strength of deposited SD3 weld metal is approximately 462MPa. The Young's modulus of the parent material is $209 \mathrm{GPa}$ and its yield ( $0.2 \%$ proof) strength at room temperature is $421 \mathrm{MPa}$. This part of the study was conducted on $5 \mathrm{~mm}$ thick sections extracted from the ends of the plate, as shown in Figure 1(a). The cut surfaces were examined particularly at the start, the middle and end portions of the cut, as shown in Figure

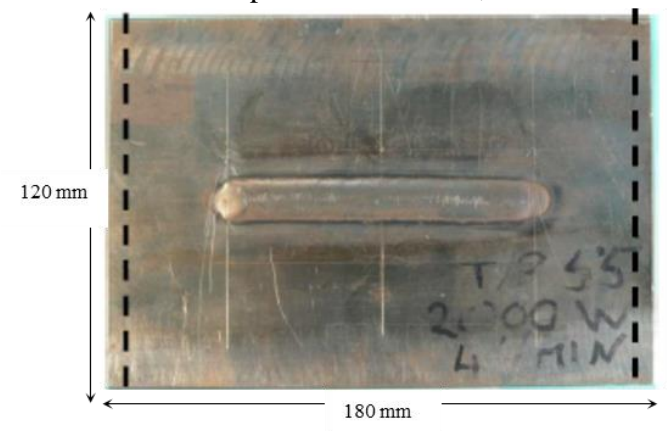

(a)
2 (a). These were far from the weld and therefore expected to be almost free of residual stresses. They were used to characterise the quality of the cut surfaces in terms of the form, surface and sub-surface damage (thickness of the recast layer due to the WEDM process, material properties changes and the depth in which residual stresses are introduced due to the WEDM process).

\section{A Ferritic Three Pass Groove Welded Plate}

A plate of SA508 Grade 3 Class 1 steel, from a pressure vessel forging, was machined to the dimensions of $200 \times 150 \times 20 \mathrm{~mm}$, as shown in Figure 1(b). A groove $200 \mathrm{~mm}$ long and $6 \mathrm{~mm}$ deep was then machined along the centre-line of one face on the plate. Three stringer beads were deposited in stacked formation in the groove. The plate was welded an unrestrained condition, being simply tacked to the bench at each end in the centre below the groove. The Young's modulus of the parent material was $209 \mathrm{GPa}$ and its yield strength at room temperature was $421 \mathrm{MPa}$. For this study, a $5 \mathrm{~mm}$ thick section was extracted from one end of the plate by using WEDM. In order to examine the quality of the WEDM cut surface, its profile was measured using a coordinate measuring machine (CMM). It has a nominally flat contour, which is free from wire entry and exit effects. This WEDM cut surface was used for the 3D surface profile scan to investigate aspects of the surface topography such as surface texture and surface roughness. This investigation was performed at the start, middle and end of the cut.

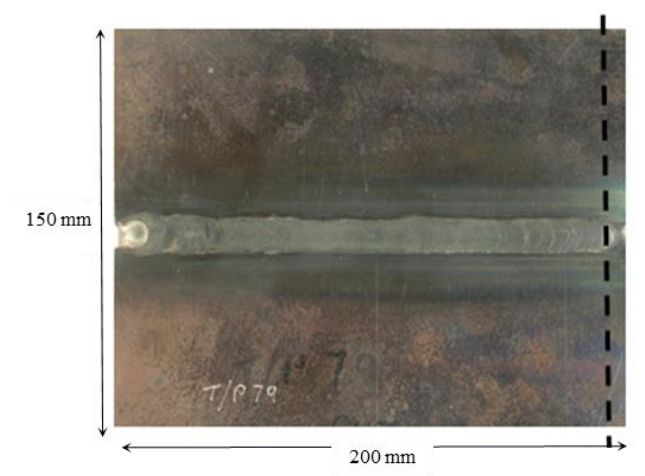

(b)

Figure 1. (a) Ferritic BoP and (b) Ferritic three pass groove welded plate, showing the positions of the $5 \mathrm{~mm}$ thick cut slices.

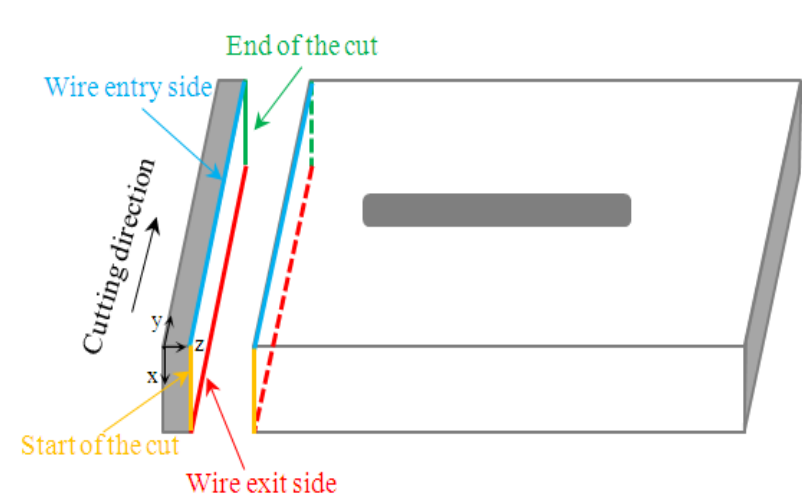

(a)

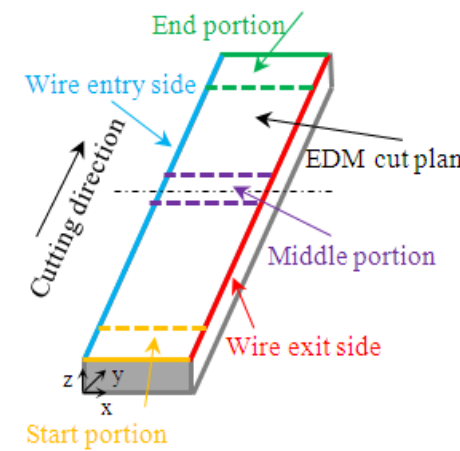

(b)

Figure 2. (a) Schematic drawing of the BoP with labelled edges of the cut part (b) The cut part showing the cut plan, start portion, middle portion and end portion.

\section{The WEDM Cuts}

An Agie Charmilles FI $440 \mathrm{ccS}$ wire Electrical Discharge Machine (WEDM) was used to perform all the cuts, including those made to extract the $5 \mathrm{~mm}$ slices from the BoP specimen. The cuts were made using $0.25 \mathrm{~mm}$ diameter brass wire. The profiles of the cut surfaces were measured using a Mitutoyo Crysta Plus 547 CMM. For this measurement, a $3 \mathrm{~mm}$ diameter Renishaw PH10M touch trigger probe was used. The three spatial coordinates of each point on a $0.5 \mathrm{~mm}$ square grid pattern on the cut surface were measured.

\section{Topography of the WEDM contour cut surfaces}

The surface profile of the first cut slice on the BoP is shown in Figure 3(a). This figure includes all the CMM data, so that at each increment in the cutting direction, the vertical axis represents the surface profile 
(contour) of the cut surface across the width of the plate. The peak-tovalley height variation of the surface profile for the first cut is approximately $20 \mu \mathrm{m}$ and also, curved edges are found at the wire entry side. Therefore, the quality of this first cut was considered unsatisfactory. In order to improve the quality of the surface profile, a second WEDM cut was made, creating a $5 \mathrm{~mm}$ wide specimen from the other end of the BoP. The second cut was performed using a modified set of WEDM cutting parameters.

The surface profile of the second cut on the BoP is shown in Figure 3(b). The map of this WEDM cut surface reveals that a cutting artefact

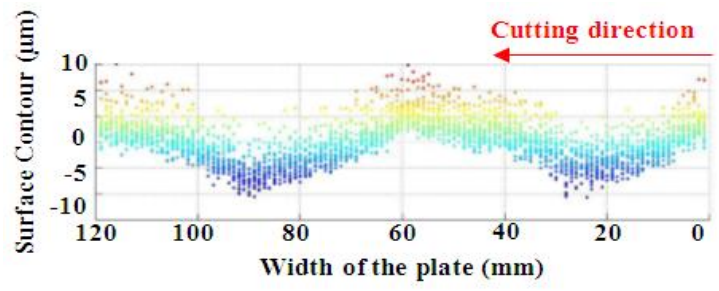

(a)

Figure 3. Surface profile (a) 1st cut (slice) on ferritic BoP; (b) 2nd cut (slice) on ferritic BoP.

\section{Surface roughness}

The surface roughness on the WEDM cut surface for the BoP was investigated at the start and the middle of the WEDM cut using a Taylor-Hobson Surtronic 3+ roughness measurement system. For each position, three longitudinal (parallel to the cutting direction) and three transverse (perpendicular to the cutting direction) measurements were made for each roughness coefficient and the results presented in Table 1 are the mean of these measurements.

Table 1. Results of roughness longitudinal profile measurements (parallel to the cutting direction) and transverse (perpendicular to the cutting direction) in two different regions of the cut surface.

\begin{tabular}{|l|l|l|l|l|l|l|}
\hline \multirow{2}{*}{ Position } & $\begin{array}{l}\text { Orientation } \\
\mathbf{w} / \mathbf{r} \\
\text { cutting } \\
\text { direction }\end{array}$ & $\mathbf{R}_{\mathbf{a}}$ & $\mathbf{R}_{\mathbf{q}}$ & $\mathbf{R}_{\mathbf{z}(\mathrm{DIN})}$ & $\mathbf{R}_{\mathbf{y}}$ & $\mathbf{S}_{\mathbf{m}}$ \\
\cline { 3 - 7 } & $(\boldsymbol{\mu m})$ & $(\boldsymbol{\mu m})$ & $(\boldsymbol{\mu m})$ & $(\boldsymbol{\mu m})$ & $(\boldsymbol{\mu m})$ \\
\hline $\begin{array}{l}\text { At start } \\
\text { of the cut }\end{array}$ & Longitudinal & 2 & 2.6 & 14 & 16 & 73 \\
\cline { 2 - 7 } $\begin{array}{l}\text { At } \\
\text { middle } \\
\text { of the cut }\end{array}$ & Transverse & 2 & 2.5 & 15 & 18 & 81 \\
\cline { 2 - 7 } & Trangitudinal & 2 & 2.5 & 14 & 15 & 73 \\
\hline
\end{tabular}

The 2D surface roughness measurement results reveal that, at the positions of start and middle of the cut, the value of $R_{a}$ (average roughness), $R_{q}$ (Root-mean-square of the peak heights), $R_{Z(D I N)}$ (peak to valley heights), $R_{y}$ (largest peak to valley height) in the transverse direction to the cut (parallel to the wire) are very similar to those in the direction parallel to the cut. The wavelength of the roughness is given by the $S_{m}$ (mean spacing between profile peaks) parameter. $S_{m}$ is constant all the way along the surface when measured in a direction parallel to the cut. Some variation of this parameter is seen in the direction perpendicular to that of the cut. Therefore, there was no

(b) of the cut.

\section{Investigation of Recast layer} increasing to $10.8 \mu \mathrm{m}$ at the end (see Figure 4). some of its parameters. in the form of a step in the surface was present, approximately $20 \mathrm{~mm}$ from the start of the cut. The cause of this cutting artefact has not yet been identified. No instability in the machine's cutting parameters was observed during the cut at this particular location. However, the peakto-valley height variation for the second WEDM cut is very small. It is worth noting that high deformations at the wire entry sides for both the cutting slices were observed. These are believed to be due to compressive surface stresses for this particular test specimen [35]. The second cut surface was found to be closer to the ideal WEDM cut. This surface was therefore chosen for the micro level investigation of an WEDM cut surface.

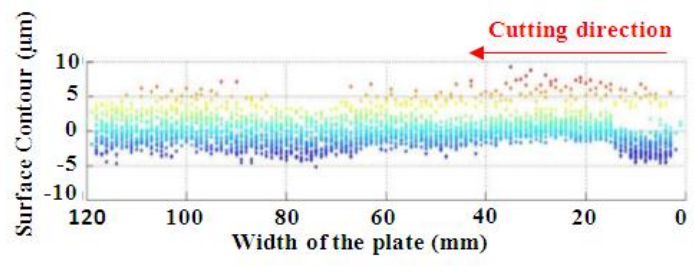
significant variation observed in roughness coefficients $R_{a}, R_{q}$, $R_{Z(D I N)}$ and $R_{y}$ at the start of the cut, compared with the middle portion

The second cut surface was sectioned such that a through thickness view of the recast layer (WEDM affected layer) was obtained. This sectioning was also performed by WEDM. Cuts were made close to the start of the cut, at the centre of the cut (where the cut is most stable) and close to the end of the cut. The cross-sections were examined in a scanning electron microscope (SEM). Sample preparation consisted of polishing to a mirror finish and embedding the specimens in electrically conductive epoxy resin. SEM imaging starts from 500x magnification and gradually the magnification was increased to $1000 x$, 2000x, 5000x and 10,000x. Eleven images were also taken using 2000x magnification for each portion of the cut at $2 \mathrm{~mm}$ intervals, covering the whole $20 \mathrm{~mm}$ width of the cut. The results presented here are the mean of these measurements. The mean recast layer thickness at the start of the cut is $10.3 \mu \mathrm{m}$, reducing to $9.8 \mu \mathrm{m}$ in the middle and

SEM imaging confirmed the presence of a recast layer on the WEDM contour cut surface (see Figure 4). However, the heat affected zone (HAZ) for each portion was not identified. It was also observed that the thickness of the recast layer is variable. The data appear to show a slight trend for the recast layer to be at its thickest near the ends of the cut. This could be caused by a variation in the WEDM spark energy as the cut progresses. This is possible because the machine monitors the process continually and automatically makes small adjustments to

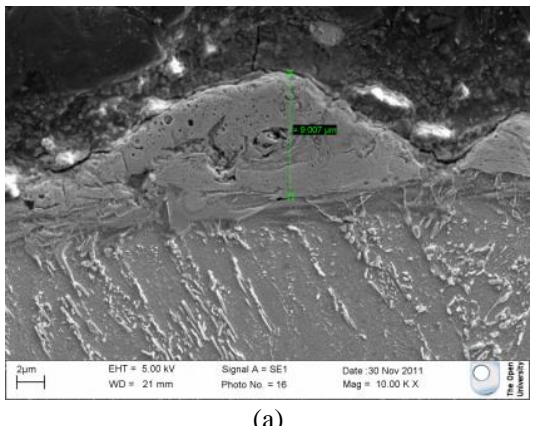

(a)

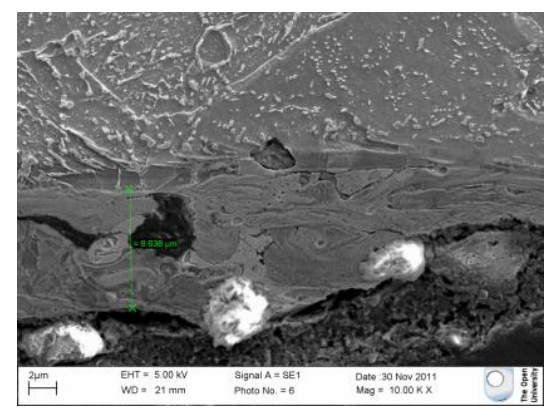

(b)

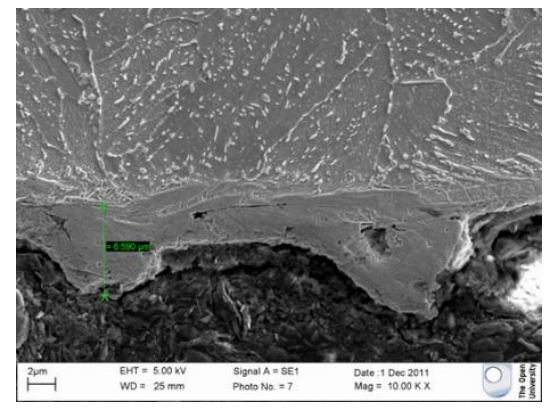

(c)

Figure 4: SEM images showing the presence of recast layer at (a) the start, (b) the middle and (c) the end of the EDM cut. 


\section{Surface Residual Stresses Induced by WEDM Cutting}

The possibility that the WEDM cutting process might induce residual stresses on the cut surface to an extent that affects the quality of the residual stress measurement merits investigation. This study presents the results of X-ray diffraction measurements of the surface residual stresses on WEDM cut specimens. If the stresses of interest are those produced by carburizing or heat treatment, it is advisable to electrochemically polish (ECP) the surface of the sample to remove the heat-affected layer [36]. Electrolytic polishing methods are particularly appropriate because they are quick, simple, highly reproducible and impart no stress on the sample surface. In order to obtain valid X-ray diffraction data, it is necessary to electro-polish specimens. In the present work, the portable MoviPol-5 tool was employed for electro polishing. This allows the user to electro-polish $9 \mathrm{~mm}$ diameter circular regions of a surface. The depth and flatness of the polished surface were measured using an optical microscope with a graduated $\mathrm{Z}$ axis control at high magnification (short depth of field). After $X$-ray diffraction measurement, the same region was then further electro-polished and re-measured. By repeating this process several times, it was possible to incrementally remove the surface and obtain a depth profile of the surface residual stress.

\section{Incremental Electro Polishing on the WEDM Surface and Measurement of Residual Stress}

Incremental electro polishing was performed through the WEDM affected layer on the central region of the second cut on the BoP specimen. A profile of the longitudinal residual stresses was obtained from the centre of the electropolished disc. Measurement was by an XSTRESS 3000 X-ray diffractometer (Stresstech Oy, VALAKOSKI, Finland) using a chromium Cr K- $\alpha, 2.2897 \AA$, 270W source and a 3 $\mathrm{mm}$ diameter collimator. The position of the peak arising from $\{211\}$ diffracting planes was at a $2 \theta$ angle of approximately $156.1^{\circ}$ [37]. The $\sin ^{2} \psi$ technique was applied using 10 scans with a range of $-45^{\circ} \leq \psi \leq$ $45^{\circ} \mathrm{psi}$ angles in order to determine the error margins for each stress measurement. Results are shown in Figure 5.

Since the contour cut surfaces were extracted from a location some distance from the weld, very low residual stresses were expected. However, the results show that high tensile stresses were present at the WEDM cut surface and these stresses must have been the result of the formation of the recast layer (see Figure 5). After the first electro polishing step, a significant value of tensile stresses was measured, these progressively decreased with depth below the original surface, until at a depth of $6 \mu \mathrm{m}$, the stress reversed to become compressive. In this present work, the incremental longitudinal stress measurements were carried out to a depth of $21 \mu \mathrm{m}$. Compressive stresses were measured in the zone extending from 6 to 21 micrometres below the surface (see Figure 5). The presence of compressive stresses at a depth of a few microns can be a response of balancing out the tensile stress in the recast layer.

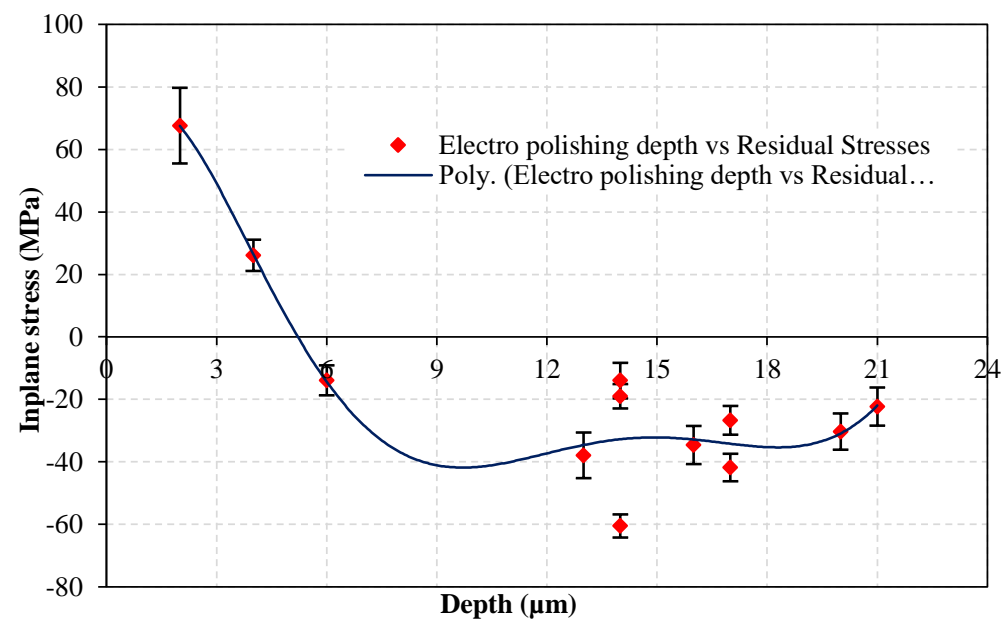

Figure 5: Electro polishing depth and measured stresses.

\section{Investigation of the changes in material properties caused by WEDM cutting}

A nano-indentation survey was performed on the same cross-section samples that were used for SEM analysis at the start, middle and end positions of the WEDM cut. Nano-indentations were made in zones that extended as close as possible to the surface of the recast layer. The hardness and elastic modulus values near the edge of the start, middle and end of the WEDM cut (within the recast layer) are shown in Table 2.

Table 2: Influence of the WEDM cutting process on hardness and elastic modulus of the workpiece.

\begin{tabular}{|l|l|l|l|l|}
\hline & Start & Middle & End & $\begin{array}{l}\text { Base } \\
\text { Material }\end{array}$ \\
\hline Hardness (GPa) & 2.34 & 2.52 & 2.33 & 1.961 \\
\hline Elastic modulus (GPa) & 153 & 200 & 167.4 & 209 \\
\hline
\end{tabular}

The materials properties were shown to have been modified up to a distance of $35 \mu \mathrm{m}$ below the WEDM cut surface. The hardness values near the edge of the start, middle and end of the EDM cut are $2.34 \mathrm{GPa}$, $2.52 \mathrm{GPa}$ and $2.33 \mathrm{GPa}$ respectively. The material has a lower hardness near the start and at the end of the cut compared with the middle portion of the cut. Correspondingly, the material has also a reduced elastic modulus near the start and end of the cut, with values of $153 \mathrm{GPa}$ and $167 \mathrm{GPa}$ respectively compared with $200 \mathrm{GPa}$ near the middle of the cut. The published figure for the elastic modulus of the ferritic parent material of the BoP specimen is $209 \mathrm{GPa}$. Thus, it can be concluded that the material affected by WEDM cutting has undergone a reduction in its elastic modulus, particularly at the start and end of the cut. This variation along the cut may also be caused by variations in the spark energy.

\section{Surface topography of WEDM cut surfaces}

The surface texture and surface roughness of the WEDM cut surface for three pass grove welded plate was measured using a confocal microscope with 50x magnification. The surveyed areas were the start and middle of the cut, over the whole width of the sample, as shown in 
Figure 6. A lateral spatial resolution of $3.3 \mu \mathrm{m}$ was achieved by stitching data sets together. The surface roughness $S_{a}$ (arithmetic mean of the absolute height) at the start of the cut was found to be $2.78 \mu \mathrm{m}$ and in the middle, $2.69 \mu \mathrm{m}$. The root-mean-square, $S_{q}$, at the start of the cut is $3.59 \mu \mathrm{m}$ and in the middle is $3.46 \mu \mathrm{m}$. These are relatively small differences, and could be accounted for by random variations within the sampled areas. However, the increased roughness near the start of the cut relative to the middle, is consistent with the picture that the other results are building, of a cutting process that is more severe at the beginning and end than in the middle of the cut.

(a)

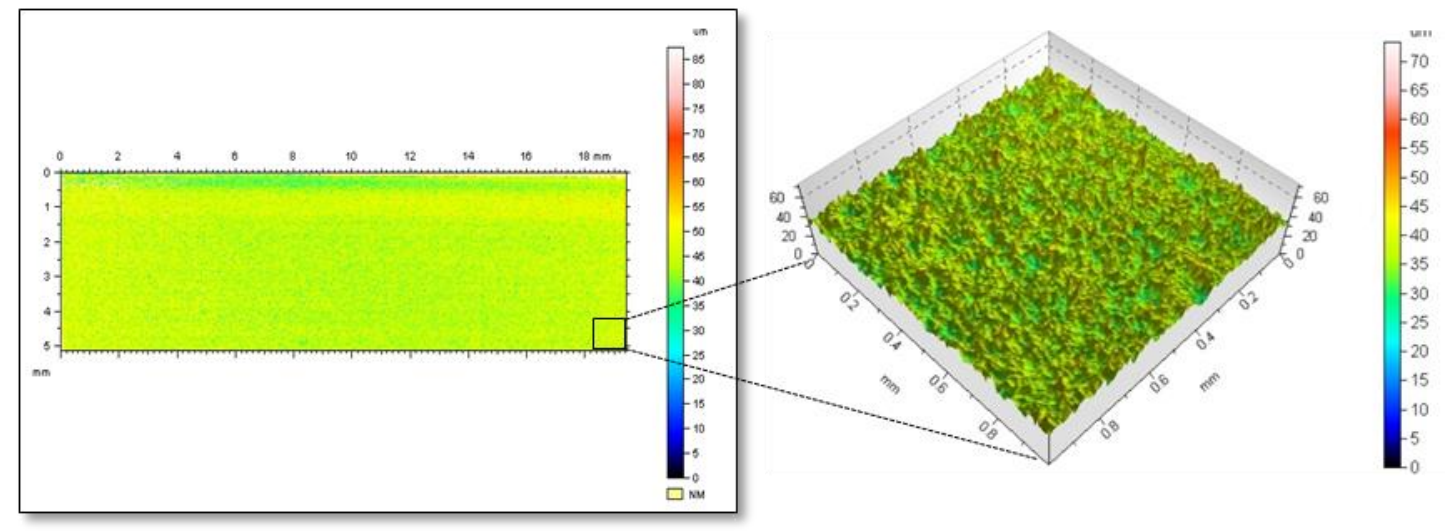

(b)

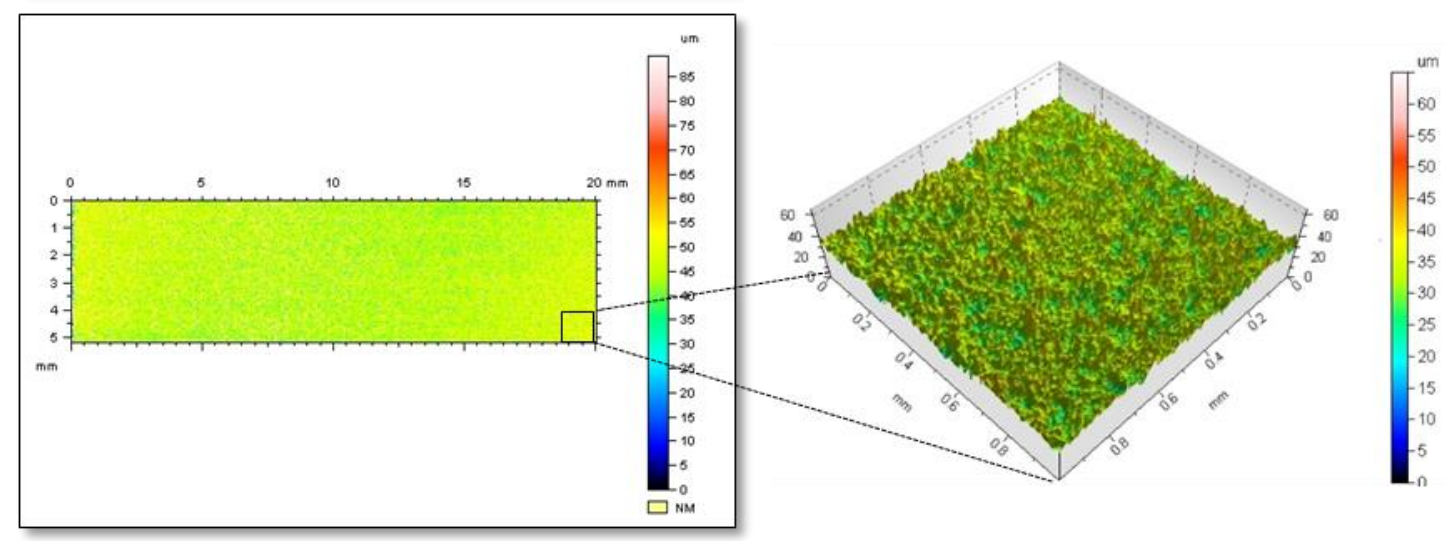

Figure 6: 2D surface profile for (a) start and (b) middle portions of the contour cut surface with 3D surface profile of a $1 \mathrm{~mm} \times 1 \mathrm{~mm}$ section.

\section{D stress analyses for contour cut surface}

The surface profile of the second cut on the BoP was used to quantify the normal component of residual stresses on the contour cut surface. A cutting artefact in the form of a step in the surface, approximately $20 \mathrm{~mm}$ from the start of the cut can be seen. High deformations were also observed at the wire entry sides. These are due to compressive surface stresses for this particular test specimen as explained here in [35].

Table 3. Represents the stress values at the start, the middle and the end portion of the contour cut surface.

\begin{tabular}{|l|l|l|l|}
\hline \multirow{2}{*}{ Portions } & $\begin{array}{l}\text { Stresses } \\
(\mathbf{M P a})\end{array}$ & $\begin{array}{l}\text { Displacements } \\
(\boldsymbol{\mu \mathbf { m } )}\end{array}$ & $\begin{array}{l}\text { Position from } \\
\text { top of the } \\
\text { plate } \\
(\mathbf{m m})\end{array}$ \\
\hline \multirow{2}{*}{ Start } & 18.5 & 1.23 & 10 \\
\cline { 2 - 4 } & -22.6 & 0.48 & 12 \\
\hline \multirow{2}{*}{ Middle } & 34 & 0.6 & 7 \\
\cline { 2 - 4 } & -34 & 0.24 & 9 \\
\hline \multirow{2}{*}{ End } & 22 & 1.6 & 13 \\
\cline { 2 - 4 } & -15 & 0.5 & 12 \\
\hline
\end{tabular}

Apart from these features the peak-to-valley height variation for the second contour cut is very small. The whole topography of the contour cut surface was examined and cut surface data were averaged along the length of the cut (the Y direction). Also, the contour cut surfaces were studied particularly at the start, the middle and at the end portions of the cut. In order to obtain the surface line profiles particular for these regions of the cut, the cut surface data were averaged along the $20 \mathrm{~mm}$ length of the cut across the start, the middle and the end of the cut portions. 2D plane stress FE models were built and respective single line profiles were applied as boundary conditions. Figure 7 represents the FE stress results along the cut length. Figure 8 and Table 3 represents the FE stress results of through-thickness line profiles and shows the stress variation within the start, the middle and the end portions of the contour cut surface.

Figure 7 represents the FE stress results along the cut length and showed that $45.5 \mathrm{MPa}$ stresses were measured $10 \mathrm{~mm}$ away from the start of the cut due to a symmetric displacement of approximately 1.7 $\mu \mathrm{m}$ due to a step cutting artefact within this region. Figure 8 and Table 3 represent the FE stress results of through-thickness line profiles and shows the stress variation within the start, the middle and the end portions of the contour cut surface. All three positions within contour cut surface clearly show a high value of compressive stress, around $220 \mathrm{MPa}$ close to the top edge of this particular test specimen (at wire entry side). For this particular case approximately $5 \mu \mathrm{m}$ contour displacement contributed in generating the $220 \mathrm{MPa}$ compressive stresses. Moreover, the stress distribution for the start portion of the contour cut surface also showed high tensile stresses of $114 \mathrm{MPa}$ up to 
$7 \mathrm{~mm}$ from top of the plate. It is different as the middle and the end portions of the cut which are because of the step cutting artefact within this region as shown in Figure 3. Cut surface features within contour surface and at the edges of the contour cut surface are significantly affected on stress propagation especially when stresses close to the surface are of interest such as in contour method. Therefore, it is very crucial to avoid these contour features during the contour cut. In addition to this, apart from ends (edges) where the quality of the cut was significantly affected by the cutting process, only the intermediate portion of the cut is considered. The WEDM cut surface still represented stress variations, these were for the start, the middle and the end portions about 18.5 MPa to $-22.6 \mathrm{MPa}, 34 \mathrm{MPa}$ to $-34 \mathrm{MPa}$ and $22 \mathrm{MPa}$ to $-15 \mathrm{MPa}$ respectively (see Table 3 ). It showed that after avoiding all cutting artefacts the specimen still represented the stress variations. These stress variations cause uncertainties in the contour method results. Therefore, it can be concluded that the range of micro level changes on the WEDM contour cut surface discussed in this study is a one of the important reasons of causing uncertainties in macro level stress measurement using contour method. Hence, it is vital to minimise micro level changes along with the cutting artefacts and unwanted features of the contour cut to reduce the uncertainties in macro level stress results.

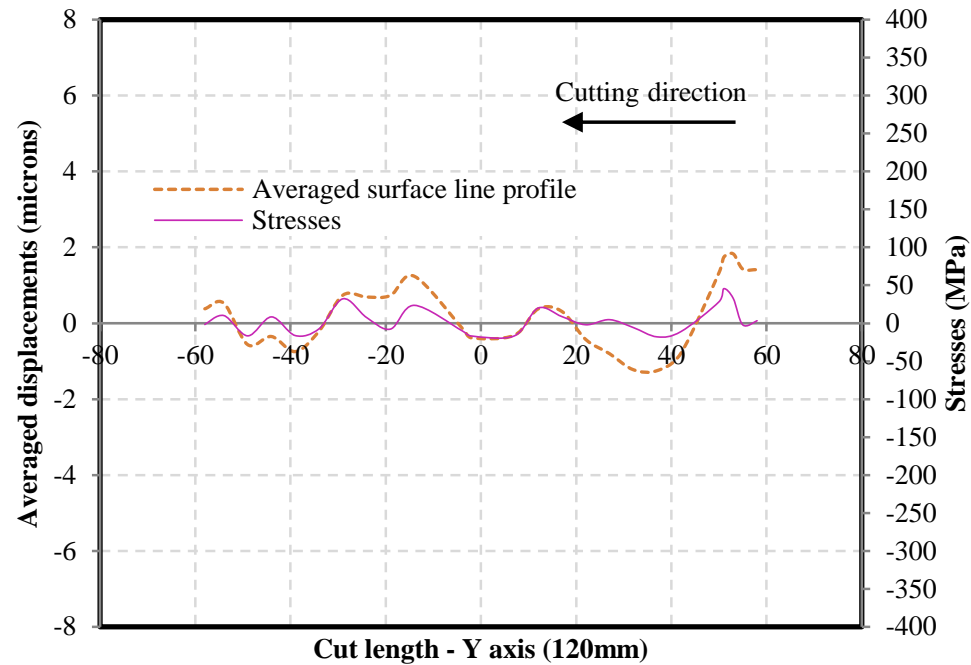

Figure 7. Represents association of distribution of the averaged displacements along the length of the cut (the Y-direction) and stresses.

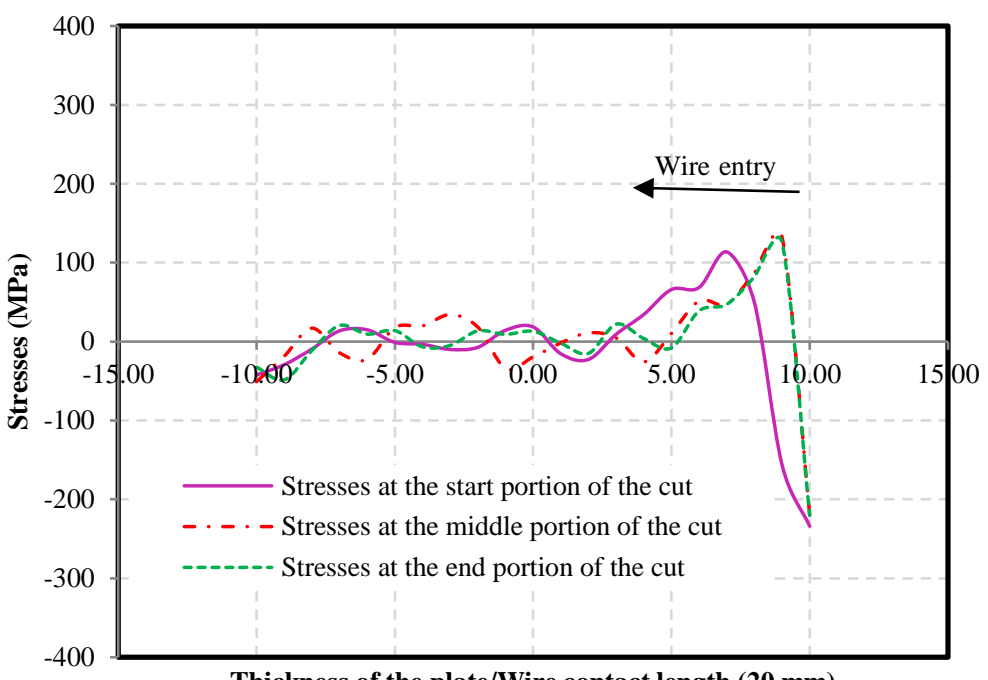

Thickness of the plate/Wire contact length $(20 \mathrm{~mm})$

Figure 8. Represents the comparison of the stress distributions through the thickness of cut with stresses particularly at the start, the middle and at the end portion of the cut. 


\section{Conclusion}

Contour cut surfaces on ferritic steel were examined to investigate the influence of the WEDM cutting process on micro level characteristic changes. surface. This study includes the measurements of the hardness and elastic modulus, the surface form and roughness of the cut, the depth of the recast layer, and the depth profile of residual stresses induced by cutting process. During this study, the micro-level changes were compared with $2 \mathrm{D}$ stress analyses results in order to determine the extent to which they affect the determination of the true level of residual stress. In the present work the WEDM cut surfaces have been studied at three different positions: close to the start of the cut; at the middle of the specimen (where the cut is most stable), and close to the end of the cut. This study reveals that during WEDM cutting the uppermost layer of the specimen is damaged. In addition, there is evidence of sub-surface changes, extending to a depth of several micrometres.

\section{Micro Scale Changes}

The study confirmed that a recast layer is formed due to the WEDM cutting process.

- $\quad$ The depth profile of the recast layer is non-uniform across the width and throughout the length of the cut.

- Due to the formation of the recast layer, in-plane tensile stresses are induced on the WEDM cut surface and these stresses decrease with distance from the top surface. The stresses turn into compression at $6 \mu \mathrm{m}$ distance from the top surface. The presence of these compressive stresses at a depth of a few microns can be a response of balancing out the tensile stress in the recast layer.

The thickness of the recast layer varies slightly along the cut. The start and end portions of the cut surfaces are more affected by the WEDM cutting processes and have a thicker recast layer than the middle portion.

- The WEDM cutting processes also affect the material properties beneath the surface, and this effect is greater at the ends of the cut than in the middle.

\section{Macro Scale Changes}

- $\quad$ Cut surface features within contour surface and at the edges of the contour cut surface are significantly affected on stress propagation especially when stresses close to the surface are of interest. Therefore it is vital to avoid these contour features during the contour cut.

- $\quad$ The surface properties at micro level on WEDM cut surface and the associated sub-surface region are not uniform along the length of the cut. Through WEDM cutting, the contour surface always suffers some surface and sub-surface changes at a scale of several micrometres. They can be affected on the residual stress measurement using the contour method.

Thus, this study showed that the good quality of the contour cut surface (achieved through controlling all described cutting features during the cutting process) still represents a notable amount of stress uncertainties in contour method results. During this particular study, the micro level changes on the contour cut surface during WEDM cutting process were clearly observed and discussed in detail. Therefore, it can be concluded that the range of these micro level changes on the contour cut surface can be affected on macro level stress measurement and caused uncertainties in the contour method stress results. Therefore, in order to minimise these uncertainties in contour method stress results, it is very crucial to reduce these micro level effects during the WEDM cutting process.

\section{Acknowledgements}

This work is carried out at The Open University Materials Engineering Laboratories. Author would like to thank Rolls Royce for supplying the specimens. I would also like to thank my senior colleagues at the
Open University to do valuable discussion on my work. I also would like to thank Pete Ledgard and Stan Hiller for their support and assistance in undertaking all experimental work. I am also grateful to the East Midlands Development Agency, Rolls-Royce Power Engineering Ltd and The Open University to provide fund for this research.

\section{References}

[1] M. Prime and A. Gonzales, "The contour method: simple 2-D mapping of residual stresses," Los Alamos National Lab., NM (US), 2000.

[2] M. B. Prime, "Cross-sectional mapping of residual stresses by measuring the surface contour after a cut," Trans.-Am. Soc. Mech. Eng. J. Eng. Mater. Technol., vol. 123, no. 2, pp. 162 168,2001

[3] F. Hosseinzadeh, A. H. Mahmoudi, C. E. Truman, and D. J. Smith, "Application of Deep Hole Drilling to the Measurement and Analysis of Residual Stresses in Steel Shrink-Fitted Assemblies," Strain, vol. 47, no. s2, pp. 412-426, 2011.

[4] G. S. Schajer, G. Roy, M. T. Flaman, and J. Lu, "Hole-drilling and ring core methods," Handb. Meas. Residual Stress., pp. 534, 1996.

[5] M. B. Prime and M. R. Hill, "Residual stress, stress relief, and inhomogeneity in aluminum plate," Scr. Mater., vol. 46, no. 1, pp. 77-82, 2002.

[6] G. S. Schajer and M. B. Prime, "Use of inverse solutions for residual stress measurements," J. Eng. Mater. Technol., vol. 128, no. 3, pp. 375-382, 2006.

[7] M. B. Prime and A. L. Kastengren, "The contour method cutting assumption: error minimization and correction," in Experimental and Applied Mechanics, Volume 6, Springer, 2011, pp. 233-250.

[8] P. Pagliaro, M. B. Prime, H. Swenson, and B. Zuccarello, "Measuring multiple residual-stress components using the contour method and multiple cuts," Exp. Mech., vol. 50, no. 2, pp. 187-194, 2010.

[9] N. S. Rossini, M. Dassisti, K. Y. Benyounis, and A. G. Olabi, "Methods of measuring residual stresses in components," Mater. Des., vol. 35, pp. 572-588, 2012.

[10] Y. S. Liao, J. T. Huang, and Y. H. Chen, "A study to achieve a fine surface finish in Wire-EDM," J. Mater. Process. Technol., vol. 149, no. 1, pp. 165-171, 2004.

[11] K. Ojha, R. K. Garg, K. K. Singh, and others, "MRR improvement in sinking electrical discharge machining: a review," J. Miner. Mater. Charact. Eng., vol. 9, no. 08, p. 709, 2010.

[12] M. Kunieda, B. Lauwers, K. P. Rajurkar, and B. M. Schumacher, "Advancing EDM through fundamental insight into the process," CIRP Ann.-Manuf. Technol., vol. 54, no. 2, pp. 64-87, 2005.

[13] W. Tebni, M. Boujelbene, E. Bayraktar, S. B. Salem, and others, "Parametric approach model for determining electrical discharge machining (EDM) conditions: effect of cutting parameters on the surface integrity,” Arab. J. Sci. Eng., vol. 34, no. 1c, pp. 101-114, 2009.

[14] M. B. Prime, "Cross-Sectional Mapping of Residual Stresses by Measuring the Surface Contour After a Cut," J. Eng. Mater. Technol., vol. 123, no. 2, pp. 162-168, Nov. 2000.

[15] P. J. Bouchard, P. Ledgard, S. Hiller, and F. Hosseinzadh Torknezhad, "Making the cut for the contour method," presented at the 15th International Conference on Experimental Mechanics, Porto, Portugal, 2012.

[16] F. Hosseinzadeh, J. Kowal, and P. J. Bouchard, "Towards good practice guidelines for the contour method of residual stress measurement," J. Eng., p. Online-only, 2014.

[17] R. Leach, L. Brown, and X. Jiang, "Guide to the measurement of smooth surface topography using coherence scanning interferometry-Issue 1368-6550." A National Measurement Good Practice Guide, No. 108, 2008. 
[18] M. D. Olson, A. T. DeWald, M. B. Prime, and M. R. Hill, "Estimation of Uncertainty for Contour Method Residual Stress Measurements," Exp. Mech., pp. 1-9, 2014.

[19] S. Akaslan and others, "The effect of machining parameters on tool electrode edge wear and machining performance in electric discharge machining (EDM)," KSME Int. J., vol. 16, no. 1, pp. 46-59, 2002.

[20] Y. Y. Tsai and C. T. Lu, "Influence of current impulse on machining characteristics in EDM," J. Mech. Sci. Technol., vol. 21, no. 10, pp. 1617-1621, 2007.

[21] F. Hosseinzadeh, P. Ledgard, and P. J. Bouchard, "Controlling the cut in contour residual stress measurements of electron beam welded Ti-6Al-4V alloy plates," Exp. Mech., vol. 53, no. 5, pp. 829-839, 2013.

[22] F. Hosseinzadeh and P. J. Bouchard, "Residual Stress Measurement of a Hipped Bonded Valve Seat Using the Contour Method," in ASME 2013 Pressure Vessels and Piping Conference, 2013, p. V06BT06A083.

[23] B. Ahmad and M. E. Fitzpatrick, "Minimization and Mitigation of Wire EDM Cutting Errors in the Application of the Contour Method of Residual Stress Measurement," Metall. Mater. Trans. A, vol. 47, no. 1, pp. 301-313, Jan. 2016.

[24] A. B. Puri and B. Bhattacharyya, "An analysis and optimisation of the geometrical inaccuracy due to wire lag phenomenon in WEDM,” Int. J. Mach. Tools Manuf., vol. 43, no. 2, pp. 151159, Jan. 2003.

[25] A. T. DeWald and M. R. Hill, "Eigenstrain-based model for prediction of laser peening residual stresses in arbitrary threedimensional bodies Part 1: Model description,” J. Strain Anal. Eng. Des., vol. 44, no. 1, pp. 1-11, Jan. 2009.

[26] P. Pagliaro, M. B. Prime, B. Clausen, M. L. Lovato, and B. Zuccarello, "Known residual stress specimens using opposed indentation," J. Eng. Mater. Technol., vol. 131, no. 3, p. 031002, 2009.

[27] Y. Traore, P. J. Bouchard, J. Francis, and F. Hosseinzadeh, “A novel cutting strategy for reducing plasticity induced errors in residual stress measurements made with the contour method," in ASME 2011 Pressure Vessels and Piping Conference, 2011, pp. 1201-1212.
[28] M. B. Prime, "Plasticity effects in incremental slitting measurement of residual stresses," Eng. Fract. Mech., vol. 77, no. 10, pp. 1552-1566, 2010.

[29] C.-C. Wang, H.-M. Chow, L.-D. Yang, and C.-T. Lu, "Recast layer removal after electrical discharge machining via Taguchi analysis: a feasibility study," J. Mater. Process. Technol., vol. 209, no. 8, pp. 4134-4140, 2009.

[30] G. Cusanelli, A. Hessler-Wyser, F. Bobard, R. Demellayer, R. Perez, and R. Flükiger, "Microstructure at submicron scale of the white layer produced by EDM technique," J. Mater. Process. Technol., vol. 149, no. 1, pp. 289-295, 2004.

[31] M. Manjaiah, S. Narendranath, S. Basavarajappa, and V. N. Gaitonde, "Effect of electrode material in wire electro discharge machining characteristics of Ti50Ni50-xCux shape memory alloy," Precis. Eng., vol. 41, pp. 68-77, Jul. 2015.

[32] H.-T. Lee and T. Y. Tai, "Relationship between EDM parameters and surface crack formation," J. Mater. Process. Technol., vol. 142, no. 3, pp. 676-683, 2003.

[33] M. Gremaud, "Measurement of near surface residual stresses using electric discharge wire machining," J. Eng. Mater. Technol., vol. 116, p. 1, 1994.

[34] J. C. Rebelo, M. Kornmeier, A. C. Batista, and A. M. Dias, "Residual stress after EDM-FEM study and measurement results," in Materials science forum, 2002, vol. 404, pp. 159164.

[35] H. Ramasawmy and L. Blunt, "3D surface characterisation of electropolished EDMed surface and quantitative assessment of process variables using Taguchi Methodology,” Int. J. Mach. Tools Manuf., vol. 42, no. 10, pp. 1129-1133, 2002.

[36] F. Hosseinzadeh and P. J. Bouchard, "Residual stress measurement of a ferritic bead on plate benchmark test specimen using the contour method," in ASME 2012 Pressure Vessels and Piping Conference, 2012, pp. 1201-1209.

[37] M. E. Fitzpatrick, A. T. Fry, P. Holdway, F. A. Kandil, J. Shackleton, and L. Suominen, "Determination of residual stresses by X-ray diffraction,” 2005. 